\title{
A bimodal tomographic reconstruction technique combining EDS-STEM and HAADF-STEM
}

\author{
Zhichao Zhong $^{\mathrm{a}, *}$, Bart Goris ${ }^{\mathrm{c}}$, Remco Schoenmakers ${ }^{\mathrm{b}}$, Sara Bals ${ }^{\mathrm{c}}$, K. Joost Batenburga,d \\ a Centrum Wiskunde \& Informatica, Amsterdam, The Netherlands \\ b FEI, Eindhoven, The Netherlands \\ c EMAT, University of Antwerp, Antwerp, Belgium \\ d Mathematical Institute, Universiteit Leiden, Leiden, The Netherlands
}

\section{A R T I C L E I N F O}

\section{Keywords:}

Electron tomography

Energy-dispersive X-ray spectroscopy

Chemical mapping

High angle annular dark field STEM

\begin{abstract}
A B S T R A C T
A three-dimensional (3D) chemical characterization of nanomaterials can be obtained using tomography based on high angle annular dark field (HAADF) scanning transmission electron microscopy (STEM) or energy dispersive X-ray spectroscopy (EDS) STEM. These two complementary techniques have both advantages and disadvantages. The Z-contrast images have good image quality but lack robustness in the compositional analysis, while the elemental maps give more element-specific information, but at a low signal-to-noise ratio and a longer exposure time. Our aim is to combine these two types of complementary information in one single tomographic reconstruction process. Therefore, an imaging model is proposed combining both HAADF-STEM and EDS-STEM. Based on this model, the elemental distributions can be reconstructed using both types of information simultaneously during the reconstruction process. The performance of the new technique is evaluated using simulated data and real experimental data. The results demonstrate that combining two imaging modalities leads to tomographic reconstructions with suppressed noise and enhanced contrast.
\end{abstract}

\section{Introduction}

Electron tomography (ET) is nowadays commonly used in materials science to characterize the three-dimensional (3D) structure and composition of nanomaterials starting from a tilt series of twodimensional (2D) projection images [1]. Typically, the projection images for ET in materials science are obtained using high angle annular dark-field (HAADF) scanning transmission electron microscopy (STEM) [2,3]. Images acquired using HAADF-STEM are called Z-contrast images because the projected intensity is related to the average atomic number that is integrated along the projection direction $[2,4]$. Consequently, the chemical composition can be characterized in 3D. However, when investigating heteronanostructures with small differences in $Z$, spectroscopic techniques are required to investigate the 3D distributions of the different chemical elements.

Previously, both energy dispersive X-ray spectroscopy (EDS) [5-8] and electron energy loss spectroscopy (EELS) [9-11] have been used in combination with tomographic reconstruction techniques. Both techniques require similar computational steps to produce element-specific images (elemental maps) that give the $2 \mathrm{D}$ projections of a chemical element, which also satisfy the projection requirement for tomography under certain circumstances $[8,10]$. In this study, we only focus on
EDS-STEM tomography.

HAADF-STEM tomography and EDS-STEM tomography are highly complementary techniques that each have advantages and disadvantages. The major advantage of HAADF-STEM tomography in comparison to EDS-STEM tomography is that it yields reconstructions with a relatively high signal to noise ratio (SNR). However, the reconstructed image intensities contain only aggregate information of all elements, while the EDS-STEM technique yields element-specific reconstructions. So far, HAADF-STEM has been combined with EDS-STEM in ET in terms of tilt series alignment [12], density estimation [13] or thickness estimation [14]. It is highly desirable to develop reconstruction techniques that can exploit the favorable properties of these complementary techniques simultaneously. The concept of "multimodal imaging" has been introduced in the field of medical imaging, where the data from several imaging modalities such as PET, SPECT, $\mathrm{CT}$ and MRI are combined in a single joint reconstruction procedure [15].

In this paper, we introduce the multi-modal imaging concept to ET, by proposing a novel HAADF-EDS bimodal tomographic (HEBT) reconstruction technique that simultaneously reconstructs from projection images acquired by two complementary imaging modalities. In this method, chemical elements are linked in the reconstruction

\footnotetext{
* Corresponding author.

E-mail address: zhong@cwi.nl (Z. Zhong).
} 
process but separated in the final output. The aim of our algorithm is to keep the element-specific feature of elemental maps while preserving the high SNR of Z-contrast images.

Section 2 will begin with discussing the mathematical models of HAADF-STEM tomography and EDS-STEM tomography. A new approach to link the models will be proposed and the HAADF-EDS bimodal tomographic reconstruction technique will be explained. In Section 3 and 4, we will investigate the performance of the new technique using both simulated and experimental data. In Section 5, the advantages and the outlook of HEBT will be discussed.

\section{Projection models and the reconstruction method}

\subsection{HAADF-STEM and EDS-STEM imaging models}

Suppose there are $k$ chemical elements in a specimen, we have $k$ volumetric objects as the unknowns to be reconstructed, so the distribution of each chemical element is represented by a voxel image. Images formed by HAADF-STEM and EDS-STEM are related to the density distributions of these chemical elements.

For HAADF-STEM projection images, it is known that the intensity is proportional to the number of electrons scattered at high angles. For a single atom, the number of these electrons is proportional to the scattering cross section which depends on its atomic number [16-18]. For thin-film specimens in which multiple scattering and absorption is negligible, the number of scattered electrons $p^{h}$ equals the sum of scattering cross sections of all the atoms probed by the electron beam:

$p^{h}=\sum_{e=1}^{k} \sigma^{(e)} N^{(e)}=\sum_{e=1}^{k} \sigma^{(e)} \frac{\int \rho^{(e)}(t) d t}{M^{(e)}}$,

where $e=1,2, \ldots k$ are the indices denoting the type of chemical element, $\sigma^{(e)}$ is the scattering cross section, $N^{(e)}$ is the number of atoms, $\rho^{(e)}(t)$ is the mass-thickness and $M^{(e)}$ is the atomic weight. By defining the HAADF-STEM response factor $z^{(e)}=\frac{\sigma^{(e)}}{M^{(e)}}$, the image grayscale is concisely expressed as the weighted sum of mass-thickness of all atoms:

$p^{h}=\sum_{e=1}^{k} z^{(e)} \int \rho^{(e)}(t) d t$.

For the sake of numerical computation, the volume to be reconstructed is often discretized into $N$ equally-spaced voxels. Thus, the density distribution of chemical element $e$ is written as a vector $\boldsymbol{\rho}^{(e)} \in \mathbf{R}^{N}, e=1,2, \ldots k$. The Z-contrast images used as tomographic reconstruction inputs are taken at different tilt angles, where every pixel specifically corresponds to a beam position and a tilted angle of the specimen. In total there are $\mathrm{M}$ pixels for all the tilted images. The grayscale on the $i_{t h}$ pixel is now written as an entry $p^{h}{ }_{i}$ in $\mathbf{p}^{h} \in \mathbf{R}^{M}$. Now the continuous line integral in Eq. (2) is replaced by the discrete ray-sum as:

$p_{i}^{h}=\sum_{e=1}^{k} z^{(e)} \sum_{j=1}^{N} w_{i j} \rho_{j}^{(e)}$

where the factor $w_{i j}$ is determined by the area intersected between the $i_{t h}$ ray integral and the $j_{t h}$ voxel (see Chapter 7.1 in [19]). Note that in the conventional HAADF-STEM tomography where the reconstruction models are defined by $p_{i}^{h}=\sum_{j=1}^{N} w_{i j} x_{j}$, the reconstructed quantity is actually $\sum_{e=1}^{k} z^{(e)} \rho_{j}^{(e)}$, which describes the distribution of the weighted sum of densities.

Unlike in HAADF-STEM tomography where projection images contain information about all atom types simultaneously, in EDSSTEM tomography each chemical element has its own series of tilted element-specific images, which depicts the projection of the chemical element and are usually called elemental maps (see more in [20,21] and chapter 4 in [22]). Their grayscales correspond to the photon counts of the characteristic X-ray of a chemical element. Under the thin-film approximation in which X-ray absorption and fluorescence is negligible, the characteristic X-rays counts $p^{(e)}$ for the $e_{t h}$ chemical element is proportional to the mass-thickness of this chemical element probed by the electron beam (discussed in $[22,23]$ ), which we define here as:

$p^{(e)}=\zeta^{(e)} \int \rho^{(e)}(t) d t$,

where $\zeta^{(e)}$ is the EDS-STEM response factor that characterizes how many characteristic X-ray counts are collected for a unit amount of the chemical element. Using the same notations as Eq. (3), the line integral relationship can be written in a discrete form as:

$p_{i}^{(e)}=\zeta^{(e)} \sum_{j=1}^{N} w_{i j} \rho_{j}^{(e)}$.

Based on the model, each chemical element can be characterized independently. Please note that in EDS-STEM tomography as in [5-7], the reconstructed quantity is the weighted density distribution $\zeta^{(e)} \rho_{j}^{(e)}$.

\subsection{Linking HAADF-STEM and EDS-STEM}

An obvious and internal connection between the two types of imaging techniques is that their projection images are both related to density distributions. However, the relations to density are based on different response factors $\left(z^{(e)}\right.$ and $\left.\zeta^{(e)}\right)$ which are difficult to estimate. To estimate these factors, special pure-element specimens need to be prepared and measured with extra labor and cost. Moreover, estimated factors are often not reusable since their values vary for different experimental set-ups.

Instead, we estimate the ratio of response factors $r^{(e)}=z^{(e)} / \zeta^{(e)}$, which we refer to here as the response ratio factors, to link the two types of images. They can be estimated based on the assumption that both types of images are linearly related to the projection of density distribution. To be more specific, if we replace the $\sum_{j=1}^{N} w_{i j} \rho_{j}^{(e)}$ by $\frac{p_{i}^{(e)}}{\zeta^{(e)}}$ (according to Eq. (5)) in Eq. (3), we have:

$p_{i}^{h}=\sum_{e=1}^{k} r^{(e)} p_{i}^{(e)}$,

where there are $k$ unknowns $r^{(e)}$. For $M$ pixels in the HAADF-STEM and EDS-STEM images, there is an overdetermined system of $M$ linear equations for the $k$ unknowns. By solving this system of linear equations (e.g. using the linear least squares method), we can estimate the response ratio factors. This can be done using only the tomographic projection images and without measuring extra specimens, and is the first step to incorporate HAADF-STEM and EDS-STEM in a simultaneous reconstruction process.

\subsection{HAADF-EDS bimodal tomographic reconstruction}

By making the substitution $x_{j}^{(e)}=z^{(e)} \rho_{j}^{(e)}$, the HAADF-STEM model of Eq. (3) and the EDS-STEM model of Eq. (5) can be rewritten as:

$p_{i}^{h}=\sum_{e=1}^{k} \sum_{j=1}^{N} w_{i j} x_{j}^{(e)}$,

$r^{(e)} p_{i}^{(e)}=\sum_{j=1}^{N} w_{i j} x_{j}^{(e)}$.

In a full system of equations, containing an equation for each measured value in each projection image, the above equations are written as $\mathbf{p}^{h}=\sum_{e=1}^{k} \mathbf{W} \mathbf{x}^{(e)}$ and $r^{(e)} \mathbf{p}^{(e)}=\mathbf{W} \mathbf{x}^{(e)}$. We see that both systems now have the same unknowns, the images $\mathbf{x}^{(e)}$ for all chemical elements. The unknowns $\mathbf{x}^{(e)}$ have the same unit as the intensities reconstructed from HAADF-STEM projections, but they can also be 
transformed into the quantitative distributions of the individual elements for each voxel when EDS response factors ( $\zeta$ factors) are provided.

To obtain reconstructions that are maximally consistent with both HAADF-STEM and EDS-STEM data, we should minimize the following residuals for EDS-STEM and HAADF-STEM simultaneously:

$$
\begin{aligned}
\mathbf{x}^{*}= & \underset{\mathbf{x}=\left(\mathbf{x}^{(1) T} \ldots \mathbf{x}^{(e) T} \ldots \mathbf{x}^{(k) T}\right)^{T}}{\operatorname{argmin}} \alpha^{2}\left\|\mathbf{p}^{h}-\sum_{e=1}^{k} \mathbf{W} \mathbf{x}^{(e)}\right\|_{2}^{2}+(1-\alpha)^{2} \\
& \sum_{e=1}^{k}\left\|r^{(e)} \mathbf{p}^{(e)}-\mathbf{W} \mathbf{x}^{(e)}\right\|_{2}^{2},
\end{aligned}
$$

where $0<\alpha<1$ is introduced here to balance between the HAADFSTEM and EDS-STEM terms. The square terms are weighted by $\alpha^{2}$ so that $\alpha$ corresponds to the image intensity. This weighting factor determines the weight of the HAADF-STEM term in the reconstruction process and should be chosen depending on the noise level of the elemental maps. In principle, $\alpha$ can be arbitrarily chosen between 0 and 1. However, in practice, if $\alpha$ is too small, the influence from the HAADF-STEM data will be hardly observable. Our empirical studies show that a number between 0.7 and 0.9 yields consistent results that balance the influences of the two modalities for our experimental data. In Section 3, we will discuss more about how the weighting factor influences reconstruction results.

The minimization problem in Eq. (9) can be formulated as a least squares problem:

$\mathbf{x}^{*}=\underset{\mathbf{x}}{\operatorname{argmin}}\left\|\mathbf{p}^{a}-\mathbf{W}^{a} \mathbf{x}\right\|_{2}^{2}$,

where

$$
\begin{gathered}
\mathbf{p}^{a}=\left(\begin{array}{c}
(1-\alpha) r^{(1)} \mathbf{p}^{(1)} \\
\vdots \\
(1-\alpha) r^{(e)} \mathbf{p}^{(e)} \\
\vdots \\
(1-\alpha) r^{(k)} \mathbf{p}^{(k)} \\
\alpha \mathbf{p}^{h}
\end{array}\right), \mathbf{W}^{a}=\left(\begin{array}{ccccc}
(1-\alpha) W & \ldots & \varnothing & \ldots & \varnothing \\
\vdots & \ldots & \vdots & \ldots & \vdots \\
\varnothing & \ldots & (1-\alpha) W & \ldots & \varnothing \\
\vdots & \ldots & \vdots & \ldots & \vdots \\
\varnothing & \ldots & \varnothing & \ldots & (1-\alpha) W \\
\alpha W & \ldots & \alpha W & \ldots & \alpha W
\end{array}\right) \\
\text { and } \mathbf{x}=\left(\begin{array}{c}
\mathbf{x}^{(1)} \\
\vdots \\
\mathbf{x}^{(e)} \\
\vdots \\
\mathbf{x}^{(k)}
\end{array}\right) .
\end{gathered}
$$

This least square problem can be solved using an iterative algorithm. In this paper, the widely used simultaneous iterative reconstruction technique (SIRT) [24] is adopted in the experiments. To incorporate the physical constraint that the elemental composition should not have negative values, we apply a non-negativity constraint to SIRT by thresholding negative values in every iteration. We refer to the complete method as HAADF-EDS bimodal tomography (HEBT).

The SIRT algorithm is more robust to noisy data than the common Weighted Backprojection algorithm, as it computes a weighted leastsquares solution, which effectively averages the noise over all projection angles, assuming that the noise follows a normal distribution. We point out that there are tomography reconstruction algorithms that are even more robust with respect to noisy data: (i) statistical reconstruction algorithms that model the statistical distribution of the noise and (ii) algorithms that incorporate prior knowledge such as discreteness or smoothness of the image. As the noise in the EDS data is Poisson distributed, but the noise in the resulting elemental maps follows a different distribution that is difficult to model in detail, we consider the Gaussian model to be a solid choice.

\section{Experiment design and data}

We design three experiments for different purposes. In the first experiment, the HEBT algorithm is applied to reconstruct 2D images from simulation data. In this simulation experiment, the stability of the HEBT reconstruction technique as a function of the response ratio factors can be investigated.

In the second experiment, we investigated cubic $\mathrm{Au}-\mathrm{Ag}$ nanoparticles using HEBT. As the two compositions ( $\mathrm{Au}$ and $\mathrm{Ag}$ ) are well separated in the particle and have a substantial difference in atomic number, the 3D distribution of the different chemical elements can be investigated using HAADF-STEM tomography and does not require EDS. Here, this HAADF-STEM reconstruction can be used as ground truth to compare the quality of the HEBT reconstructions in comparison to conventional EDS reconstructions.

The key advantage of HEBT with respect to conventional HAADFSTEM reconstruction only becomes clear if the HAADF-STEM reconstruction does not allow for straightforward segmentation of the elements, either because the difference in Z-contrast between the elements is low, or because the elements are mixed at a sub-voxel resolution. In such cases, HEBT can potentially reconstruct the individual 3D elemental volumes (not possible by HAADF-STEM), while achieving a more faithful reconstruction at lower noise level compared to conventional EDS reconstructions. This advantage is illustrated by the results of the third experiment, applying the HEBT algorithm to another nanoparticle in which an alloy of $\mathrm{Au}$ and $\mathrm{Ag}$ is present.

\subsection{Phantom simulation}

The first experiment is based on a 2D phantom image shown in Fig. 1, which was created to resemble a slice of the non-alloyed $\mathrm{Au}-\mathrm{Ag}$ nanoparticle (see Fig. 3). Fig. 1(a) and (b) are the Au and Ag phantom objects with homogeneous density. Fig. 1(c) is a Z-contrast phantom image of $\mathrm{Au}$ and $\mathrm{Ag}$ phantoms weighted by HAADF-STEM response factors that are assumed to be $z^{(A u)}=79^{1.5} \mathrm{~kg} / \mathrm{m}^{2}$ and $z^{(A g)}=47^{1.5} \mathrm{~kg} / \mathrm{m}^{2}$ [16]. To simulate projection images, tilt series of projections were computed using the ASTRA toolbox [25]. The projection geometry has 512 pixels and 31 tilt angles from $-75^{\circ}$ to $75^{\circ}$ with a step size of $5^{\circ}$.

The HAADF-STEM sinogram (Fig. 1(f)), which is assumed to be low-noise, is simply assigned as the tilt series of the Z-contrast phantom. For EDS-STEM, two sinograms (Fig. 1(d) and (e)) were generated by applying Poisson noise to the tilt series of $\mathrm{Au}$ and $\mathrm{Ag}$ phantom objects. The EDS-STEM mapping process was simulated in a way that the X-ray count on each pixel is rendered as a random integer generated from the Poisson distribution. Based on the EDS-STEM models, the mean parameters of the Poisson distributions were assigned as the tilt series multiplied by the response factors. The response factors were selected as $\zeta^{(A u)}=1.88 \times 10^{-2} \mathrm{~kg} / \mathrm{m}^{2}$ and $\zeta^{(A g)}=2.4 \times 10^{-2} \mathrm{~kg} / \mathrm{m}^{2}$ so that the mean expected numbers of X-ray counts approximate the mean X-ray counts in the elemental maps of the first sample (Fig. 3(b) and (c)). A filtering operation using an 8pixel 1D Gaussian filter was applied to the EDS-STEM sinograms as an easy implementation of noise smoothing $[8,26]$. The intensity of the HAADF-STEM sinogram is at a much larger order of magnitude than the EDS-STEM sinograms.

\subsection{Au-Ag nanoparticles}

For the real-world experiments, tilt series of projection images were acquired using the same procedures for both $\mathrm{Au}-\mathrm{Ag}$ samples. First, the sample was mounted on the tomographic holder placed in an electron microscope (Tecnai Osiris, FEI company) equipped with four silicon drift detectors (SuperX system, FEI company). During the tilt series, the sample was tilted from $-75^{\circ}$ to $75^{\circ}$ with a tilt increment of $5^{\circ}$ for the first sample. At each tilt, a Z-contrast image was first recorded by the HAADF detector. The sample was then scanned with an acquisition time of $300 \mathrm{~s}$ to record X-rays spectrum images over 2048 energy channels. In order to reduce the shadowing effect of SDD detectors, the 


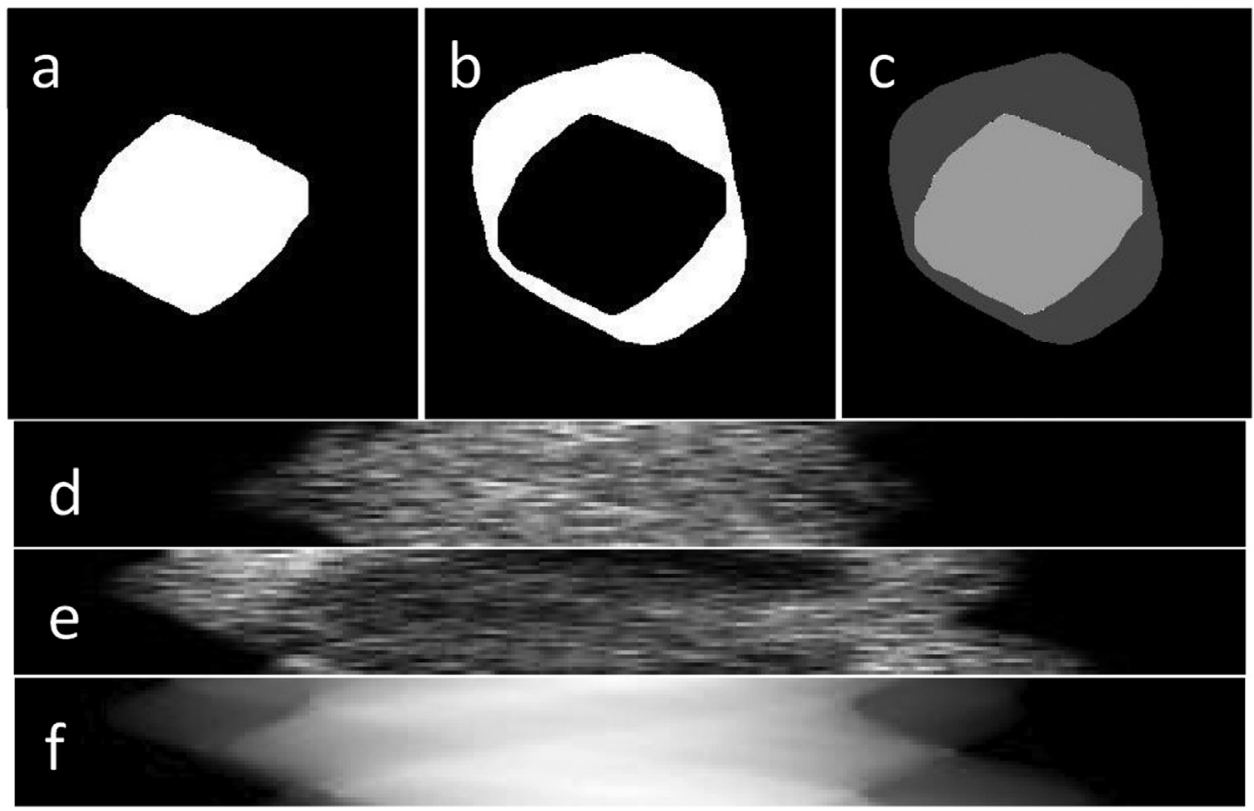

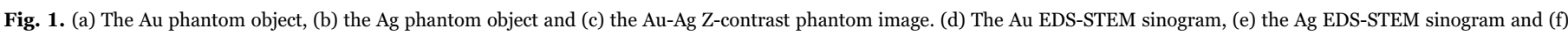
the HAADF-STEM sinogram.

detectors on one side to which X-rays were blocked were turned off, while the other two detectors on the other side were turned on [27]. A tilt series of the second sample was acquired using almost the same procedures except that the sample was tilted over 29 steps from $-70^{\circ}$ to $70^{\circ}$.

The raw data were then processed before being used as tomographic reconstruction input data. For HAADF-STEM, the tilt series of Z-contrast projection images were aligned using the cross-correlation method. The intensity damping has also been corrected by linearizing the nonlinear intensity-thickness relation [28]. For EDS-STEM, the spectrum images were denoised using principal component analysis (PCA) decomposition/reconstruction [13,29]. The high peaks near $8.040 \mathrm{keV}$ and $8.904 \mathrm{keV}$ come from $\mathrm{Cu}$ in the holder, which will overwhelm and dominate the other components in PCA if they are included. To avoid this, we only took out the energy channels near the $\mathrm{Au}$ and $\mathrm{Ag}$ peaks for PCA decomposition (Fig. 2 (b)) (the characteristic peaks are $M_{\alpha}=2.15 \mathrm{keV}, M_{\beta}=2.20 \mathrm{keV}$ and $L_{\alpha}=9.70 \mathrm{keV}$ for Au, and $L_{\alpha}=2.98 \mathrm{keV}$ and $L_{\beta}=3.19 \mathrm{keV}$ for Ag). After PCA decomposition, we examined every component and selected the first 15 components for PCA reconstruction and abandoned the remaining components as noise. Next, the denoised spectrum images near characteristic channels were extracted and summed up to the elemental maps (Fig. 2(b)).

Note that since the X-ray counts are very low for such a high resolution, even after PCA denoising the elemental maps remain very noisy. Therefore, we applied an averaging image filter with a $12 \times 12$ pixel Gaussian kernel (rotational-symmetric) to the elemental maps. Finally, the elemental maps were again aligned to match the Z-contrast images using the cross-correlation method. For each sample, the data processing steps resulted in three tilt series of projection images for each sample: two tilt series of elemental maps and one tilt series of Zcontrast images (see examples in Fig. 3).

\section{Experimental results}

In addition to the HEBT reconstructions, we also computed HAADF-STEM tomographic reconstructions from Z-contrast projection images and EDS-STEM tomographic reconstructions from elemental maps. All the reconstructions were computed using the SIRT algorithm with non-negativity constraints unless indicated otherwise. The number of iterations is chosen to be large enough to assure convergence of HEBT as well as not too large to avoid over-fitting the least square problem. The weighting factor $\alpha$ was chosen as 0.7 unless indicated, which we found to be a good value in our experiments that balances the influence of the EDS-STEM and HAADF-STEM data. The response ratio factors used in HEBT were estimated by fitting the linear models of Eq. (6) using the non-zero pixels in the tomographic input data using the NNLS (Non-negative least squares) algorithm (see chapter 23, page 161, in [30]).

We can assess the image quality of reconstructions with reference images in the first two cases. For the simulation, we can compare reconstructions with the phantom images; for the non-alloy $\mathrm{Au}-\mathrm{Ag}$ nanoparticle, we use the segmentations acquired from the Z-contrast reconstructions as the ground-truth references. Here we use three types of image quality metrics. (i) Structural similarity index (SSIM, [31]) computes structural similarity between images, which aligns with image quality perceived by human eyes. Since image intensities are different for HAADF-STEM and EDS-STEM, we exclude the luminance and contrast terms for SSIM, and only compute the structure term. (ii) Mean-squared error (MSE) simply computes the difference between reference images $(\mathbf{x})$ and reconstructions (y) which were scaled by scaling factors that give minimal MSE. The computation is formulated as: $\operatorname{MSE}(\mathbf{x}, \mathbf{y})=\min _{c}\|\mathbf{x}-c \mathbf{y}\|_{2}^{2}$, where $c$ is the scaling factor. (iii) The difference in pixels (DP) is computed as the $l_{1}$ norm of the difference between two binary images. The reference images are already binary, while reconstructions for elements have continuous intensity. Given the knowledge that elements have homogeneous density, we binarize the reconstructions with thresholds, which are chosen as the ones giving minimal DP. Mathematically this can be written as $D P(\mathbf{x}, \mathbf{y})=\min _{b}\left\|\mathbf{x}-B_{b}(\mathbf{y})\right\|$, where $B_{b}(\mathbf{y})$ means binarizing an image with the threshold $b$.

\subsection{Phantom objects}

Estimating the response ratio factors is the first step of HEBT. The response ratio factors for $\mathrm{Au}$ and $\mathrm{Ag}$ were estimated to be $\mathbf{r}_{e s t}=\left[3.27 \times 10^{4}, 1.68 \times 10^{4}\right]$, while the ground truths are $\mathbf{r}_{g t}=\left[3.66 \times 10^{4}, 1.43 \times 10^{4}\right]$ based on the given response factors. The goodness of how the data matches the linear model is indicated by the coefficient of determination $R^{2}=0.91$, which can be interpreted as $91 \%$ of the data can be explained by the linear model. 


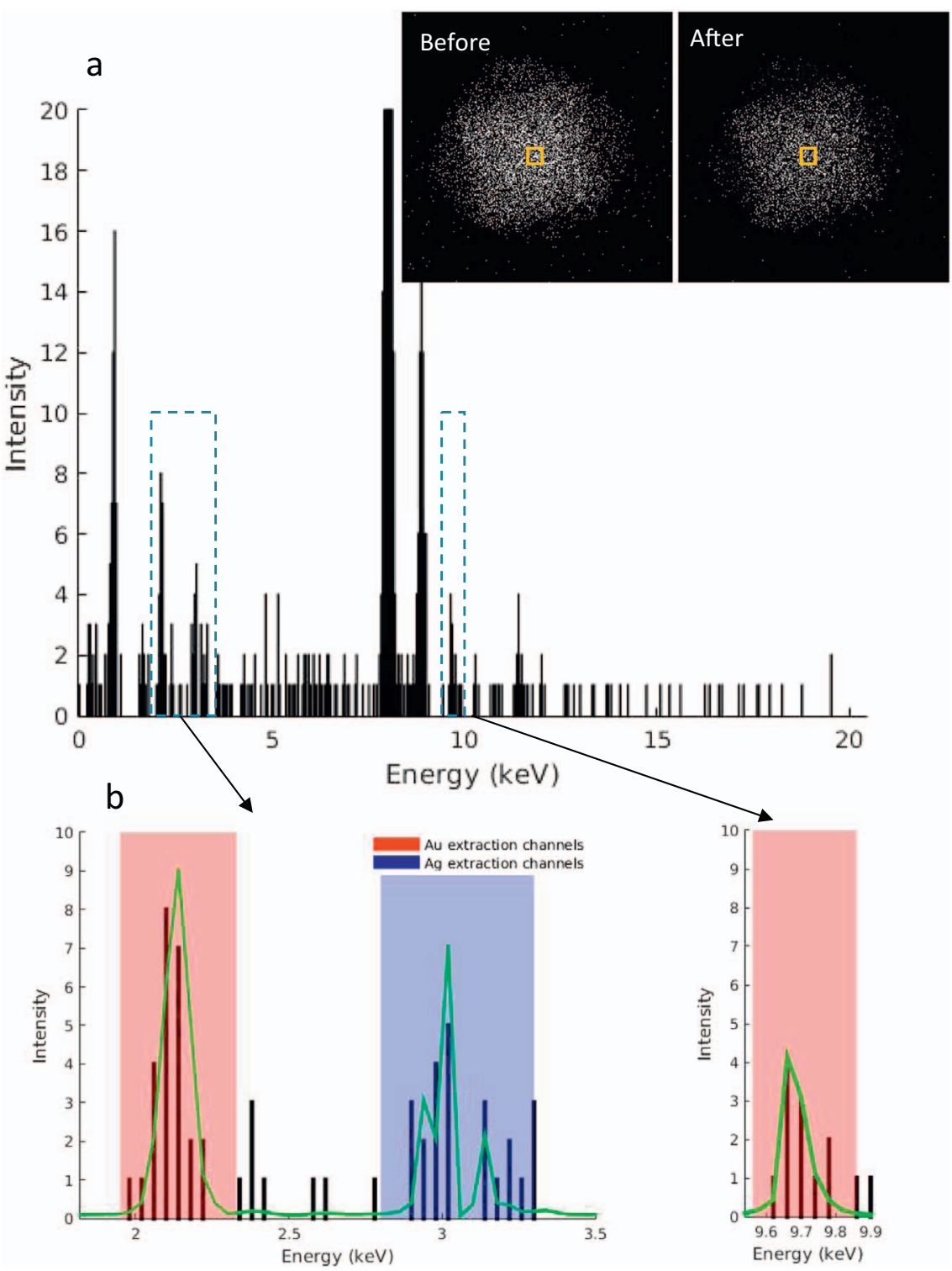

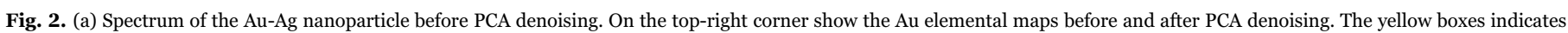

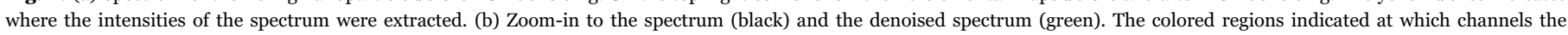
denoised spectrum images were extracted to elemental maps.

The simulation study aims at studying the stability of HEBT when errors are present in the estimated response ratio factors. Here, the estimated response ratio factors differ from the ground-truth by $-10.66 \%$ and $17.48 \%$ respectively. Thus, reconstructions were made by HEBT with estimated and ground-truth response ratio factors respectively (Fig. 4). Both were computed with weighting factor $\alpha=0.7$ and for 200 iterations. First of all, intuitively we see both results show less noise and sharper contrast compared to EDS-STEM tomographic reconstructions. Secondly, the deviation of response ratio factors only results in slightly different distributions of noise between elemental reconstructions. We can observe that 'streaks' are more suppressed in Fig. 4 (a) than (b) since $r_{A u}$ is overestimated compared to the true values. On the other hand, Fig. 4 (d) looks noisier than (e) because $r_{A g}$ is underestimated. From the image quality metrics, HEBT with $\mathbf{r}_{e s t}$ produces nearly the same image quality as HEBT with $\mathbf{r}_{g t}$. One noticeable result is that although HEBT with $\mathbf{r}_{g t}$ outperforms HEBT with $\mathbf{r}_{\text {est }}$ in terms of SSIM and DP, for MSE the result with $\mathbf{r}_{e s t}$ is better. This can be explained as that noise has been taken into account when estimating the response ratio factors, consequently yielding better statistical fitting for reconstructions. In conclusion, the HEBT algo- rithm output shows good stability $w . r . t$. errors in the estimation of the response ratios Table 1 .

\subsection{Non-alloy Au-Ag nanoparticle}

The first sample that is experimentally investigated is an $\mathrm{Ag}$ nanoparticle with a diameter of approximately $110 \mathrm{~nm}$ with an embedded $\mathrm{Au}$ octahedron. Examples of Z-contrast images and elemental maps are given in Fig. 3, indicating that $\mathrm{Ag}$ and $\mathrm{Au}$ are well separated.

The response ratio factors $r^{(A u)}$ and $r^{(A g)}$ were estimated from all the non-zero pixels using the NNLS algorithm. The fitting results are $\mathbf{r}=\left[5.31 \times 10^{4}, 8.64 \times 10^{4}\right]$ with a coefficient of determination $R^{2}=0.95$. The example of Fig. 3 (d) shows that the sum of elemental maps weighted by $\mathbf{r}$ closely but not perfectly matches the HAADFSTEM projection image due to noise. After the estimation, the reconstructions were computed slice by slice in a volume of $300 \times 300 \times 300$ voxels by solving the least square problem of Eq. (9).

Fig. 5 shows the 2D reconstruction images at different slices. Compared to EDS-STEM reconstructions, HEBT reconstructions de- 

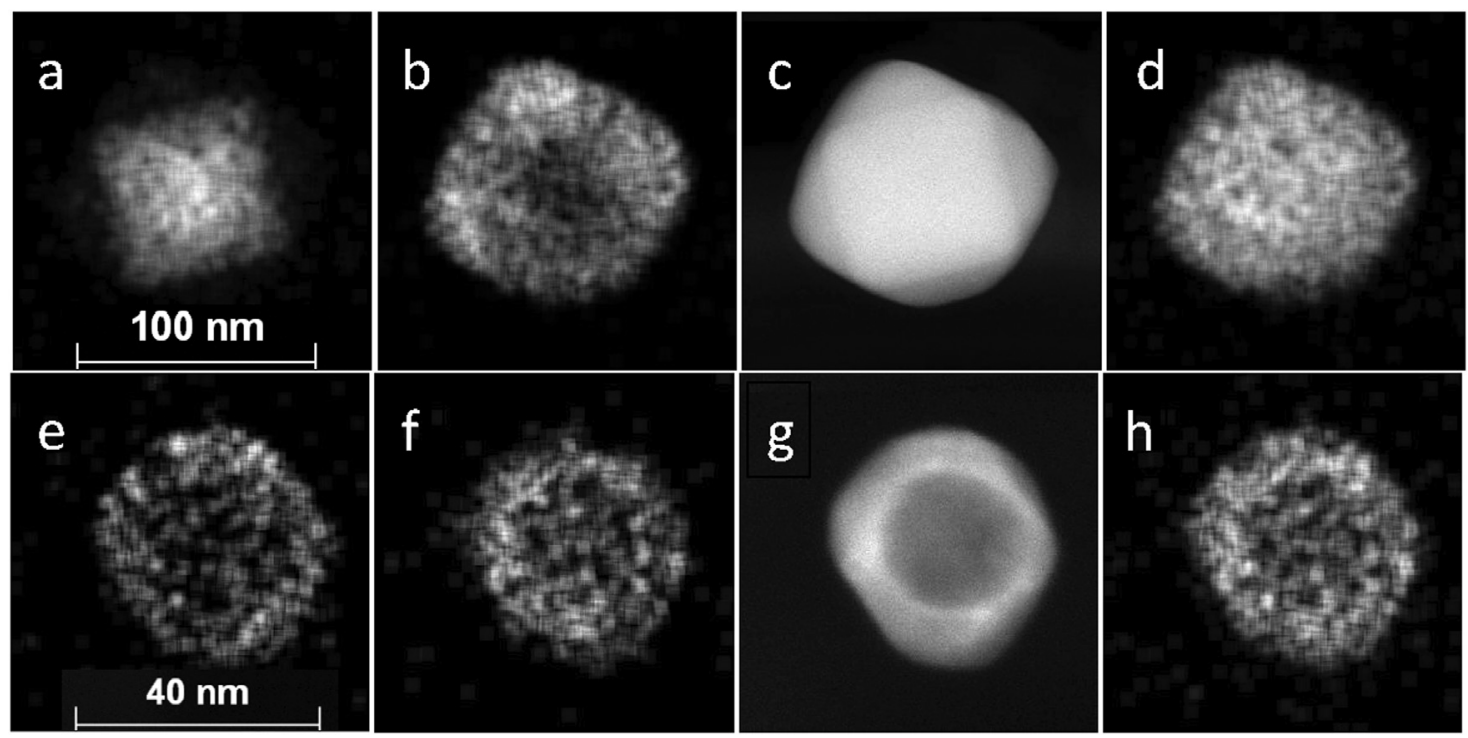

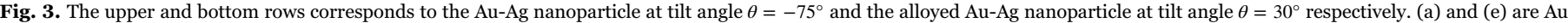

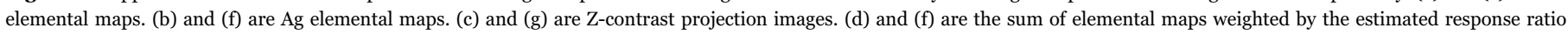
factors. The image sizes are $300 \times 300$ pixels.
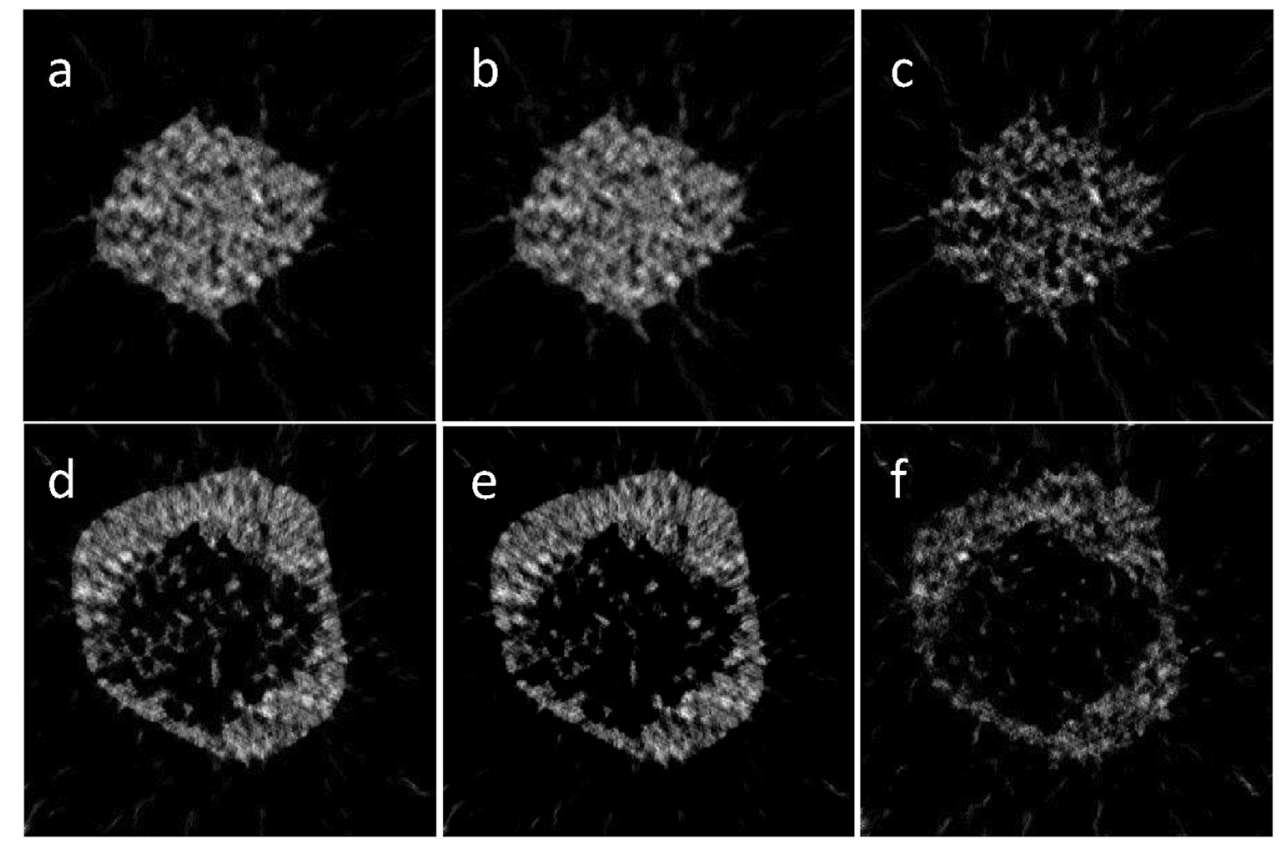

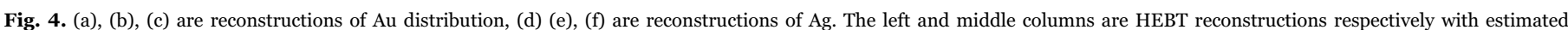
response ratio factors $\mathbf{r}_{e s t}$ and with ground-truth response ratio factors $\mathbf{r}_{g t}$; the right column is reconstructions from only EDS-STEM elemental maps.

Table 1

Image quality metrics of reconstructions.

\begin{tabular}{llll}
\hline Methods & HEBT with $\mathbf{r}_{\text {est }}$ & HEBT with $\mathbf{r}_{g t}$ & EDS-STEM \\
\hline SSIM Au & 0.9923 & 0.9917 & 0.9437 \\
SSIM Ag & 0.9837 & 0.9835 & 0.8739 \\
MSE Au & 0.0260 & 0.0260 & 0.0801 \\
MSE Ag & 0.0449 & 0.0469 & 0.0740 \\
DP Au & 1664 & 1940 & 6936 \\
DP Ag & 4915 & 4762 & 7318 \\
\hline
\end{tabular}

monstrate smoother intensity distributions, suppressed noise levels and clearer boundaries. Especially for the Ag reconstructions, morphological analysis becomes easier as exterior boundaries show a sharper contrast to the background after being regularized by the HAADF-
STEM term. The HAADF term also regularizes intensities of noise to concentrate within the particle and not spread across the background. For example, in the HEBT reconstructions for $\mathrm{Au}$, we can see noise forming a 'shadow' of the entire particle on the background. Fortunately, the 'shadow' noise is rather weak and can be removed by thresholding or smoothing.

The HAADF-STEM reconstructions have clear boundaries between $\mathrm{Au}$ and $\mathrm{Ag}$ in this case. Therefore, we can easily segment the two particles, and use the segmentation as the ground truth for reconstruction quality assessment. In Fig. 6, the HAADF-STEM reconstruction was segmented into two parts by manually recognizing the boundaries in every slice using the FEI Amira 6.0 software, which are considered as the ground truths of compositional distributions. Meanwhile, we also demonstrate the 3D volume rendering of EDS-STEM reconstructions and HAADF reconstructions for comparison. The image quality metrics 


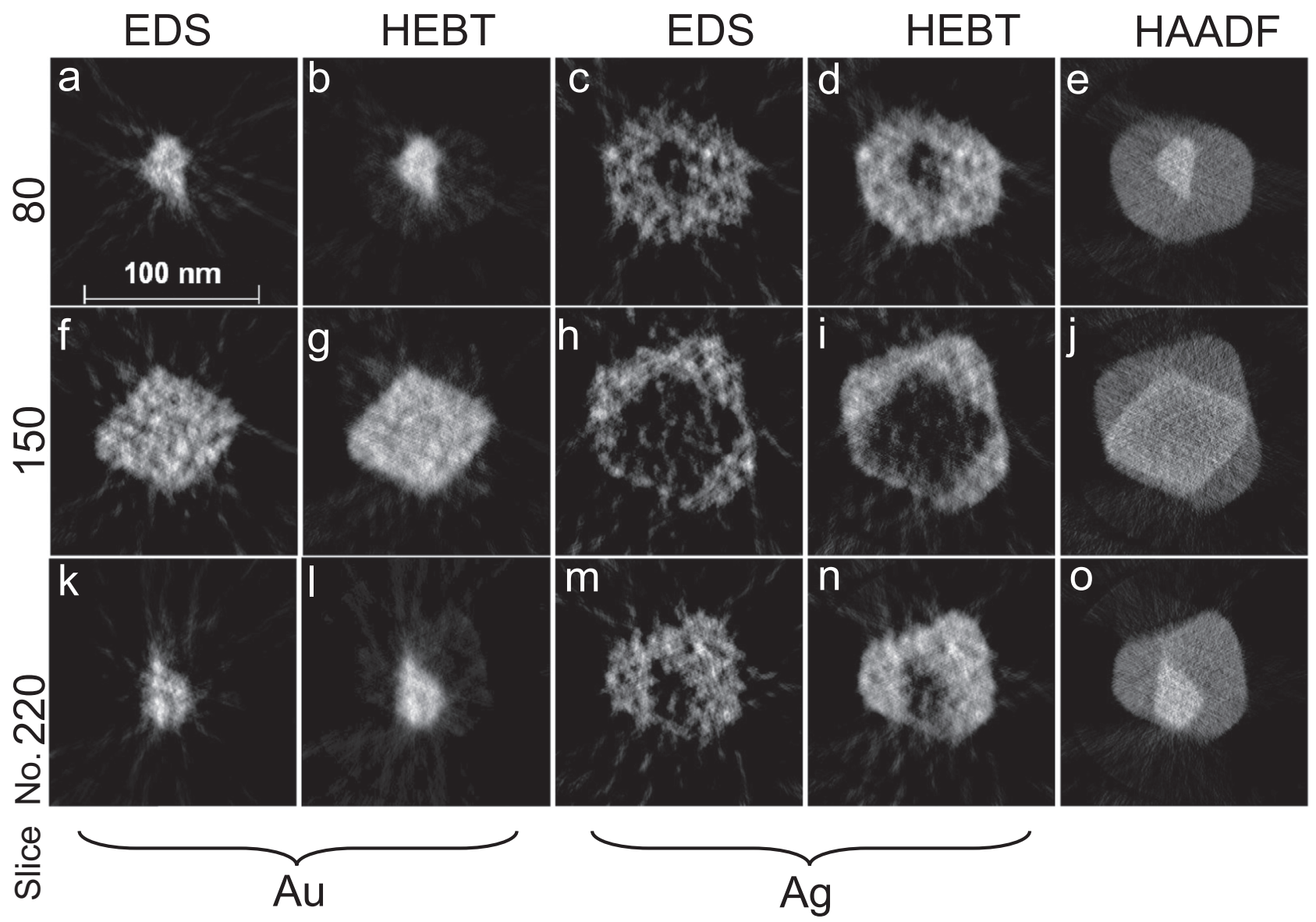

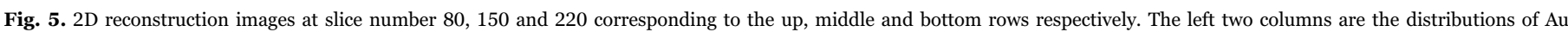

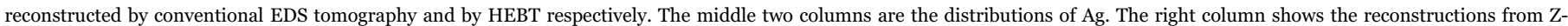
contrast projection images.

were computed in 3D and listed in Table 2. The metrics show that the image quality of HEBT reconstructions is intrinsically enhanced in comparison to conventional EDS reconstructions.

Based on the ground truth from the segmented HAADF-STEM reconstructions, the influence of two parameters for HEBT can be investigated: the weighting factor $\alpha$ and the number of iterations. Here, we sampled the weighting factor from 0.01 to 0.99 for HEBT reconstructions with different numbers of iterations for one slice. Fig. 7 plots the MSE indices at each weighting factor. It first indicates a decrease of MSE as $\alpha$ grows, as the noise is increasingly suppressed by the HAADF-STEM term. When $\alpha$ gets close to 1, MSE starts to increase rapidly after reaching a minimum. To understand this phenomenon, we plot the reconstructions at $\alpha=0.7$ for 50/100/500 iterations. It shows that for 50 iterations, $\mathrm{Ag}$ appears in the reconstruction of $\mathrm{Au}$ (Fig. 8 (b)). The explanation is that a too large $\alpha$ makes
Table 2

Image quality metrics.

\begin{tabular}{lll}
\hline Methods & EDS-STEM & HEBT \\
\hline SSIM Au & 0.9661 & 0.9680 \\
SSIM Ag & 0.9024 & 0.9097 \\
MSE Au & 0.0093 & 0.0069 \\
MSE Ag & 0.0368 & 0.0229 \\
DP Au & 233,805 & 134,861 \\
DP Ag & $1,213,822$ & 674,403 \\
\hline
\end{tabular}

minimizing residuals for EDS-STEM terms become very inefficient due to their small weights. If the residuals of EDS-STEM terms remain large while the residual of HAADF-STEM has already been minimized, backprojection from HAADF-STEM projection images will show up in
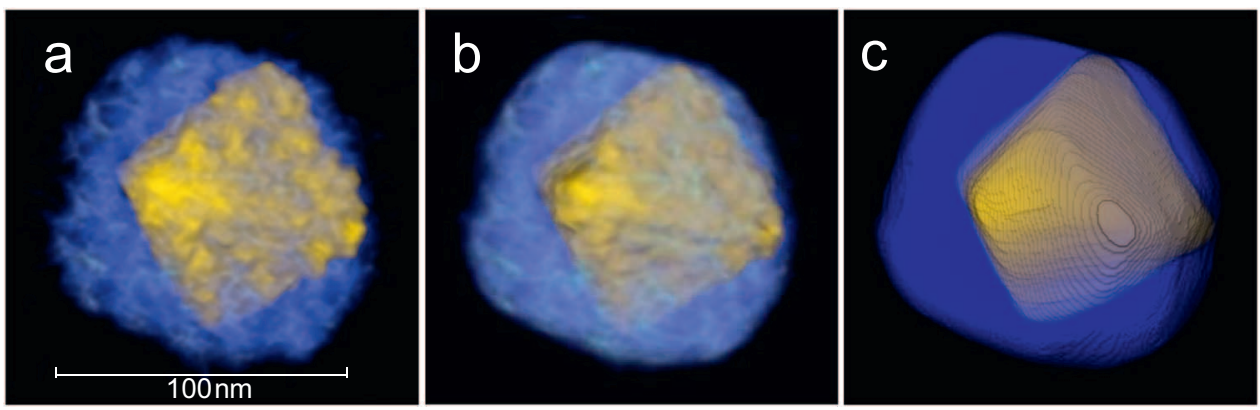

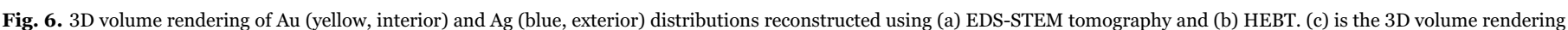
of $\mathrm{Au}$ and $\mathrm{Ag}$ segmented from HAADF-STEM reconstructions (ground-truth). 

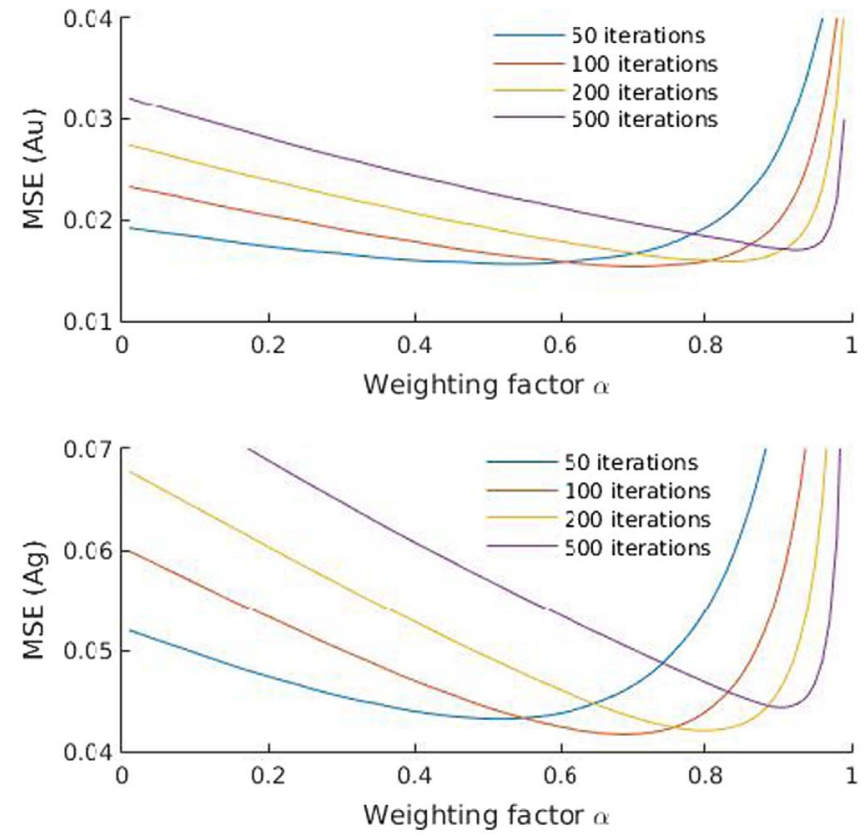

Fig. 7. Mean-squared errors for $\mathrm{Au}$ and $\mathrm{Ag}$ reconstructions under different iterations. The reconstructions are sampled for weighting factors from 0.01 to 0.99 .

the reconstructions. In such a case, we can see appearance from the other compositions. The appearance can be reduced by increasing the number of iterations. In this case, Ag disappears in the Au reconstruction as the number of iterations grows. On the other hand, if the number of iterations is chosen very large, this may lead to over-fitting of the least-square problem, which results in the presence of noise in reconstructions. The over-fitting also explains why - for small weighting factors - the MSE metric decreases as the number of iterations increases (see Fig. 7). In a word, the weighting factor influences the noise suppression and the convergence of least square problem; to guarantee convergence for large weighting factors, a large number of iterations should be adopted. From Fig. 7, we conclude that $\alpha=0.7$ and 100 iterations are close-to-optimal settings for this example.

To investigate whether HEBT leads to improved ability to spatially resolve the chemical composition of nanomaterials in comparison to separate EDS-STEM reconstructions, we have conducted two addi- tional validation experiments. In the first experiment, a binary mask is created from the HAADF-STEM reconstruction, which is then enforced during each iteration step of the SIRT reconstruction from elemental maps. For the second experiments, a binary mask is created based on the Z-contrast projection images which are subsequently applied to the elemental maps prior to tomographic reconstruction. The results of these experiments are shown in Figs. 9 (a-d). It can be seen that the results are qualitatively similar to the reconstructions without the masks in the sense that the chemical composition is no better spatially localized than in the unmasked case. This can be contrasted to Fig. 5, where the better localization is visible, clearly demonstrating the advantage of our HEBT reconstruction technique.

\subsection{Alloyed Au-Ag nanoparticle}

In this case, we demonstrate the application of HEBT on data for which 3D compositional analysis is difficult for both EDS-STEM tomography and HAADF-STEM tomography. The sample is an Au-Ag alloy nanoparticle with a diameter about $30 \mathrm{~nm}$. As suggested by the Zcontrast images in Fig. 3 (d), segmentation cannot be made based on HAADF-STEM reconstructions since no clear boundary exists between the two compositions. Although elemental distributions can be reconstructed from elemental maps, the elemental maps are very noisy (Fig. 3(e) and (f)) and lead to strong noise in the EDS-STEM tomographic reconstruction results.

The HEBT reconstructions were computed using $\alpha=0.7$ for 200 iterations. The response ratio factors were estimated to be $\mathbf{r}=\left[5.63 \times 10^{4}, 6.52 \times 10^{4}\right]$ with a coefficient of determination $R^{2}=0.79$. The values for the same elements differ from the first experimental case. This is likely due to an intensity rescaling that was applied when storing the HAADF-STEM data. As our response ratio factors are automatically scaled, this does not affect the final results.

Compared with EDS-STEM tomography, HEBT gives more interpretable results with less noise and stronger contrast to the background as shown in the 2D slices of Fig. 10. Here, since we no longer have ground-truth images, we cannot compute image quality metrics. Fig. 11 shows that the HEBT reconstructions provide more information in 3D on the concentration of the different elements compared to the EDSSTEM reconstructions. The elemental distributions with reduced noise indicate that the $\mathrm{Au}$ is more concentrated in the exterior than $\mathrm{Ag}$.
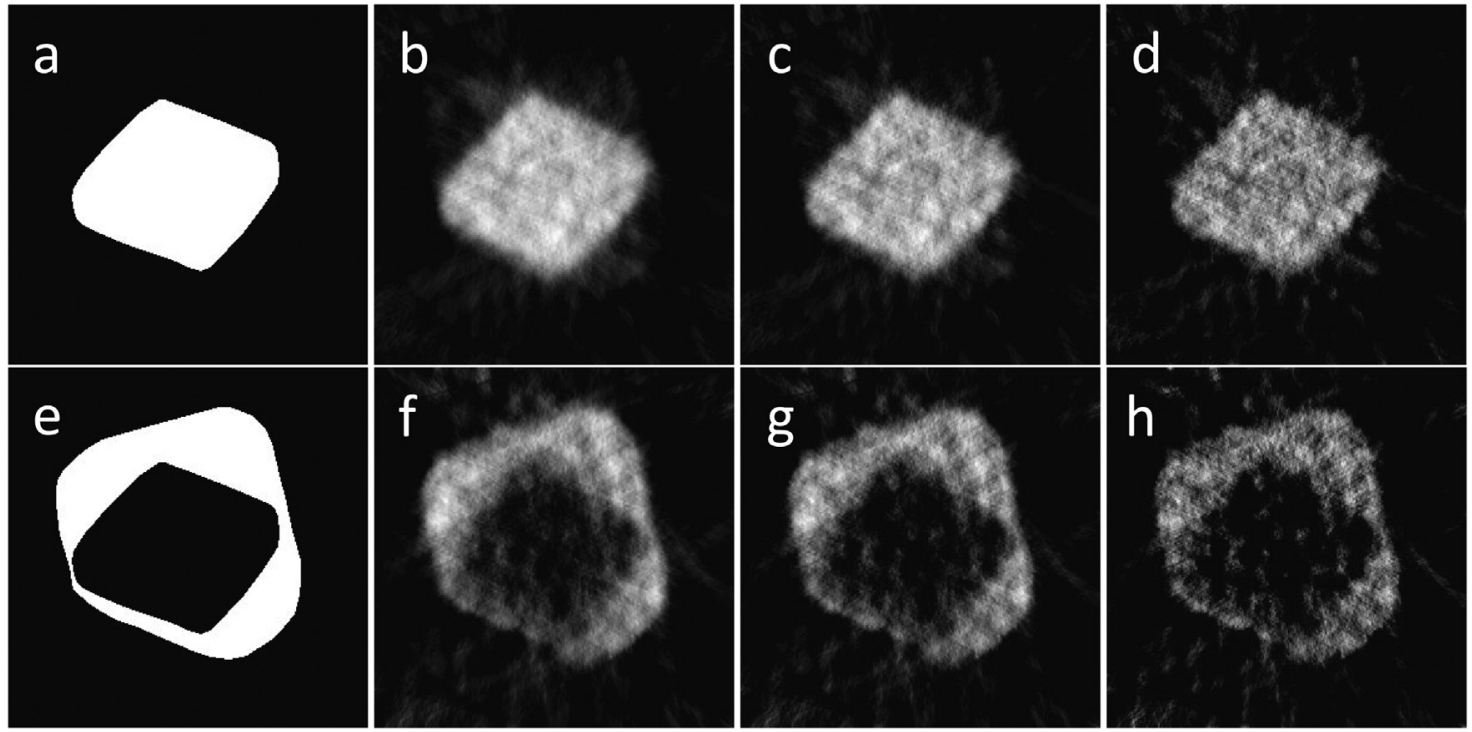

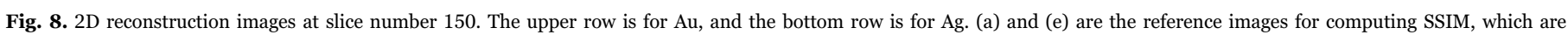
segmented from the HAADF-STEM reconstruction. (b)/(f), (c) $/(\mathrm{g})$ and (d) $/(\mathrm{h})$ are respectively HEBT reconstructions with weighting factor $\alpha=0.7$ under $50,100,500$ iterations. 

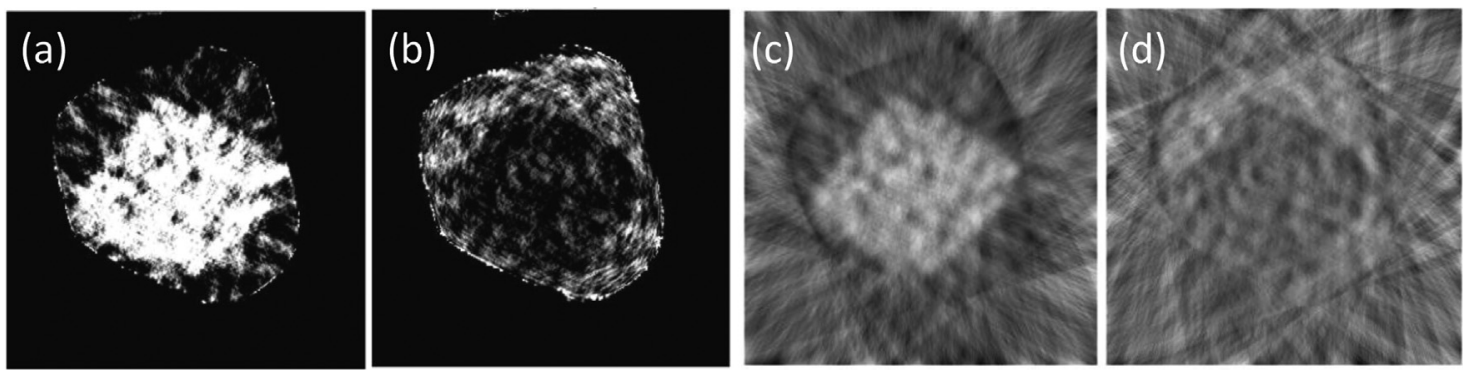

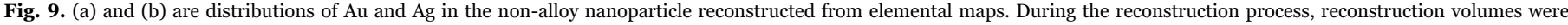

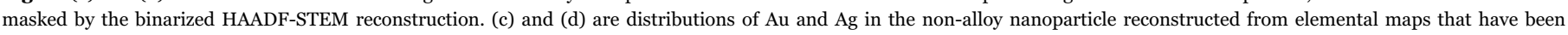
masked by binarized Z-contrast images.

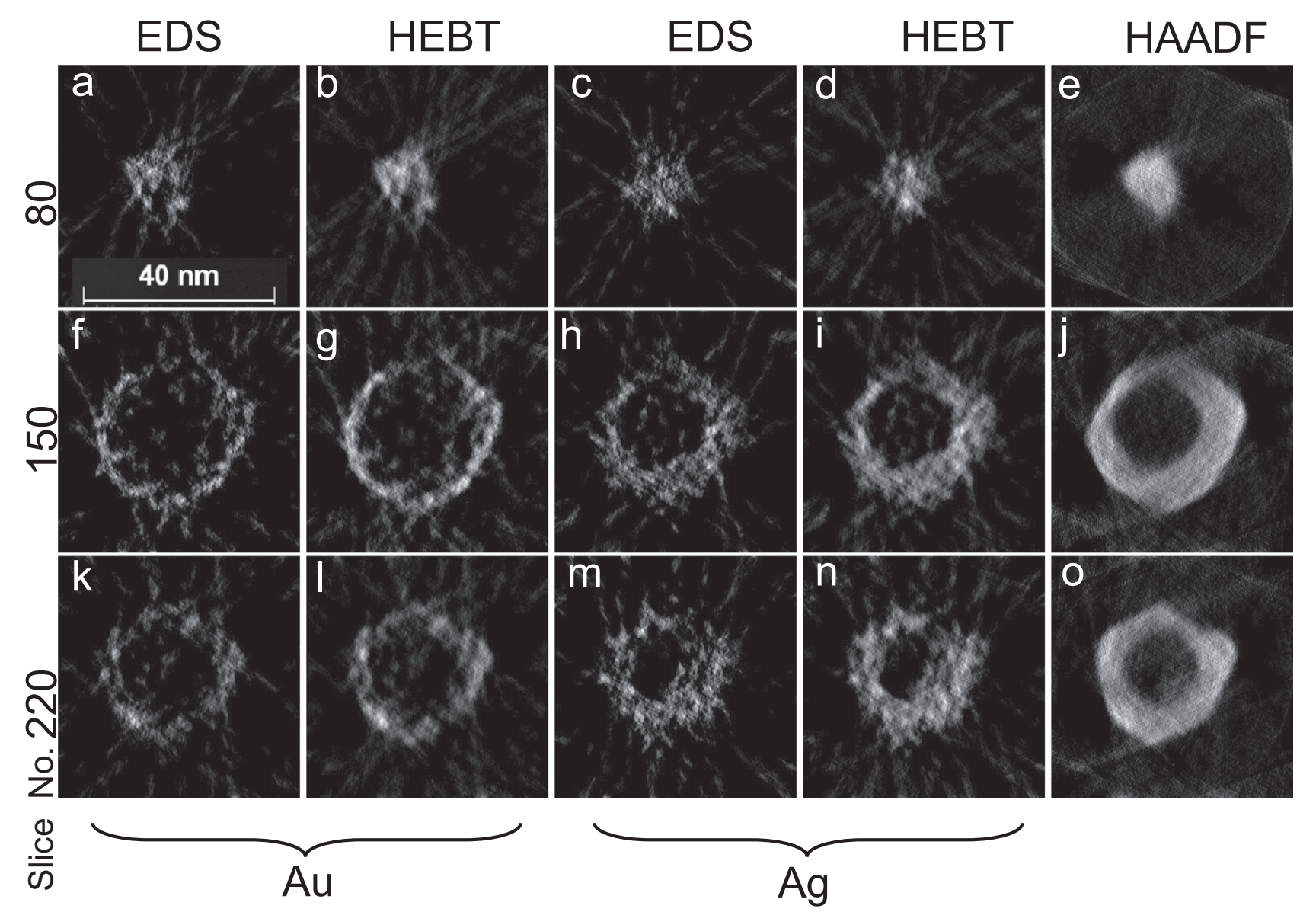

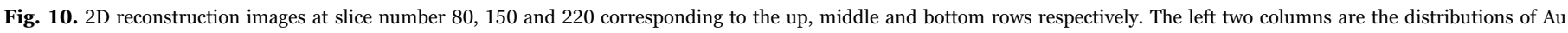

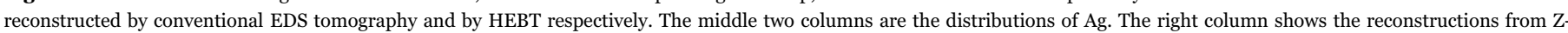
contrast projection images.
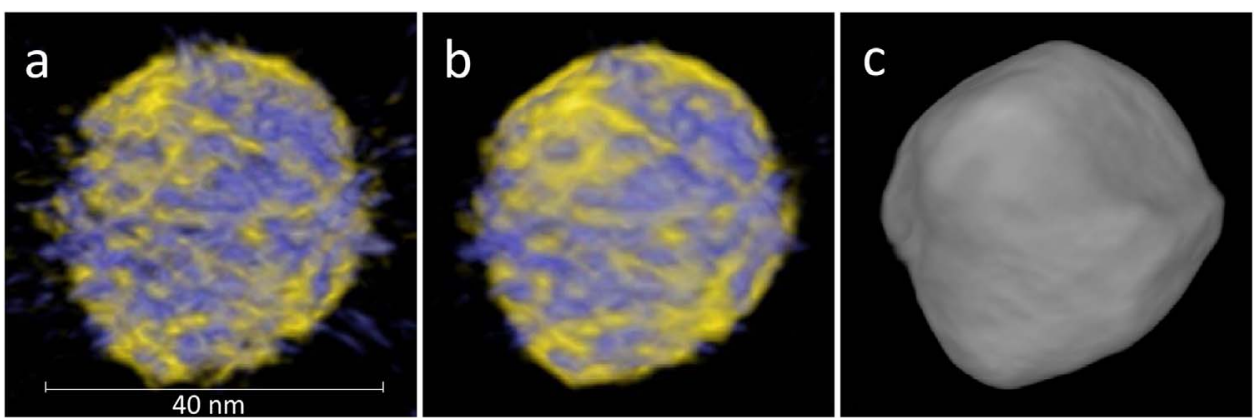

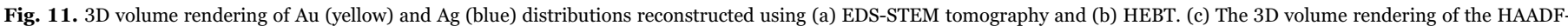
STEM reconstruction. 


\section{Conclusion}

In this study, we have developed HAADF-EDS bimodal tomography for the 3D characterization of the chemical composition at the nanometer scale. This technique first links elemental maps with Zcontrast images that are recorded simultaneously in STEM mode and contain complementary information. The linking is made by estimating response ratio factors that give the linear relation of two types of images and by scaling their intensities to the same unit. Simultaneously from two types of projection images, 3D elemental distributions are reconstructed. The reconstruction process results in a simultaneous minimization of the projection errors of both EDS-STEM and HAADFSTEM and is carried out using an iterative method such as SIRT.

HEBT has first been tested on a phantom object that is based on hetero-nanoparticles. We specifically demonstrated that HEBT is robust w.r.t. errors in the response ratio factor estimation. Subsequently, we used HEBT to reconstruct the 3D elemental distributions of two different nanoparticles. To investigate the image quality enhancement of HEBT, we first reconstructed an $\mathrm{Au}-\mathrm{Ag}$ nanoparticle where the different elements could be distinguished based on Zcontrast. Taking the Z-contrast reconstruction results as the ground truth, we see that reconstructions computed by HEBT are improved in comparison to EDS-STEM tomographic reconstructions in terms of image quality. In this case, we also demonstrated that HEBT with a large weighting factor requires a large number of iterations to converge and separate between elements. In the second experimental case, Ag and $\mathrm{Au}$ are alloyed, and thus it is impossible to investigate the 3D distributions of the chemical elements based on HAADF-STEM tomography. Using the HEBT techniques, we are able to investigate the spatial distribution of $\mathrm{Ag}$ and $\mathrm{Au}$ inside the particle. The interpretation of the final result is more straightforward in comparison to conventional EDS-STEM tomography, for which the results contain more noise.

The HEBT algorithm is based on the assumption that both HAADFSTEM projection images and EDS-STEM elemental maps can be modeled as perfect linear projections of the structure. In practice, this assumption is not completely valid as nonlinear phenomena such as Xray absorption and electron channelling may break the projection requirement $[8,13]$. In addition, the EDS noise follows a Poisson distribution, while the least squares problem in Eq. (9) is based on the assumption that the noise follows a Gaussian distribution. Our purpose here is to demonstrate the feasibility of HEBT, while recognizing these sources of inaccuracy. In future work, we plan to incorporate more sophisticated models for self-absorption (similar to [13]) and elemental map extraction (similar to [29]), as well as to adopt advanced denoising reconstruction algorithm based on the Poisson noise model such as the EM method (see Chapter 5 in [32]).

Conventionally, quantitative analysis based on EDS-STEM measurements suffer from the high noise level in these measurements. By combining EDS and HAADF, especially by imposing the 3D information obtained by HAADF, the improvement in the reconstructions (compared to pure EDS-STEM reconstruction) will lead to more reliable quantification, provided that the corresponding zeta factors are known. This application also requires an accurate estimation of the response ratio factors. Therefore, we are developing a new estimation method which is based on the Poisson noise model rather than the Gaussian noise model.

In conclusion, the newly developed HEBT technique is a promising technique to analyze chemical compositions of nanomaterials in 3D. By exploiting more complete information from two complementary types of images, it can characterize the elemental distribution even when it is not straightforward using HAADF-STEM and EDS-STEM tomography. This advantage means that the 3D characterization of chemical composition can be pushed to materials with smaller dimensions and more complex compositions.

\section{Acknowledgement}

This research is supported by the Dutch Technology Foundation STW (http://www.stw.nl/), which is part of the Netherlands Organization for Scientific Research (NWO), and which is partly funded by the Ministry of Economic Affairs, Agriculture and Innovation under project number 13314. It is also supported by the Flemish research foundation (FWO Vlaanderen) by project funding (G038116N) and a postdoctoral research grant to B.G. Funding from the European Research Council (Starting Grant No. COLOURATOMS 335078) is acknowledged by S.B. The authors would like to thank Dr. Bernd Rieger and Dr. Richard Aveyard for useful discussions, and Prof. Dr. Luis M. Liz-Marzan for providing the investigated samples. We also acknowledge COST Action MP1207 for networking support.

\section{References}

[1] G.T. Herman, Fundamentals of Computerized Tomography: Image Reconstruction from Projections, 2nd ed., Springer, London, 2009.

[2] P.A. Midgley, M. Weyland, J.M. Thomas, B.F.G. Johnson, Z-Contrast tomography: a technique in three-dimensional nanostructural analysis based on rutherford scattering, Chem. Commun. (2001) 907-908.

[3] P.A. Midgley, M. Weyland, 3D electron microscopy in the physical sciences: the development of Z-contrast and EFTEM tomography, Ultramicroscopy 96 (3) (2003) 413-431.

[4] C. Kübel, A. Voigt, R. Schoenmakers, M. Otten, D. Su, T.-C. Lee, A. Carlsson, J. Bradley, Recent advances in electron tomography: TEM and HAADF-STEM tomography for materials science and semiconductor applications, Microsc. Microanal. 11 (05) (2005) 378-400.

[5] Z. Saghi, X. Xu, Y. Peng, B. Inkson, G. Möbus, Three-dimensional chemical analysis of tungsten probes by energy dispersive x-ray nanotomography, Appl. Phys. Lett. 91 (25) (2007) 251906.

[6] A. Genç, H. Cheng, J. Winterstein, L. Pullan, B. Freitag, 3D chemical mapping using tomography with an enhanced XEDS system, Microsc. Anal. 116 (2012) 23-25.

[7] K. Lepinay, F. Lorut, R. Pantel, T. Epicier, Chemical 3D tomography of $28 \mathrm{~nm}$ high K metal gate transistor: STEM XEDS experimental method and results, Micron 47 (2013) 43-49.

[8] T.J. Slater, A. Janssen, P.H. Camargo, M.G. Burke, N.J. Zaluzec, S.J. Haigh, STEMEDX tomography of bimetallic nanoparticles: a methodological investigation, Ultramicroscopy 162 (2016) 61-73.

[9] K. Jarausch, P. Thomas, D.N. Leonard, R. Twesten, C.R. Booth, Four-dimensional STEM-EELS: enabling nano-scale chemical tomography, Ultramicroscopy 109 (4) (2009) 326-337.

[10] L. Yedra, A. Eljarrat, R. Arenal, E. Pellicer, M. Cabo, A. Lopez-Ortega, M. Estrader, J. Sort, M.D. Baro, S. Estradé, et al., EEL spectroscopic tomography: towards a new dimension in nanomaterials analysis, Ultramicroscopy 122 (2012) 12-18.

[11] L. Yedra, A. Eljarrat, J.M. Rebled, L. López-Conesa, N. Dix, F. Sánchez, S. Estradé, F. Peiró, EELS tomography in multiferroic nanocomposites: from spectrum images to the spectrum volume, Nanoscale 6 (12) (2014) 6646-6650.

[12] B. Goris, L. Polavarapu, S. Bals, G. Van Tendeloo, L.M. Liz-Marzán, Monitoring galvanic replacement through three-dimensional morphological and chemical mapping, Nano Lett. 14 (6) (2014) 3220-3226.

[13] P. Burdet, Z. Saghi, A.N. Filippin, A. Borrás, P.A. Midgley, A novel 3D absorption correction method for quantitative EDX-STEM tomography, Ultramicroscopy 160 (2016) 118-129.

[14] D. Zanaga, T. Altantzis, L. Polavarapu, L.M. Liz-Marzán, B. Freitag, S. Bals, A new method for quantitative XEDS tomography of complex heteronanostructures, Particle \& Particle Systems Characterization.

[15] H. Zaidi, M.-L. Montandon, A. Alavi, The clinical role of fusion imaging using PET, CT, and MR Imaging, Magn. Reson. Imaging Clin. North Am. 18 (1) (2010) $133-149$.

[16] M.M. Treacy, Z dependence of electron scattering by single atoms into annular dark-field detectors, Microsc. Microanal. 17 (06) (2011) 847-858.

[17] S.J. Pennycook, Z-contrast STEM for materials science, Ultramicroscopy 30 (1) (1989) 58-69.

[18] T. Walther, A new experimental procedure to quantify annular dark field images in scanning transmission electron microscopy, J. Microsc. 221 (2) (2006) 137-144.

[19] A.C. Kak, M. Slaney, Principles of Computerized Tomographic Imaging, IEEE Press, 1988.

[20] P. Schlossmacher, D. Klenov, B. Freitag, H. Von Harrach, Enhanced detection sensitivity with a new windowless XEDS system for AEM based on silicon drift detector technology, Microsc. Today 18 (04) (2010) 14-20.

[21] G. Haberfehlner, A. Orthacker, M. Albu, J. Li, G. Kothleitner, Nanoscale voxel spectroscopy by simultaneous EELS and EDS tomography, Nanoscale 6 (23) (2014) $14563-14569$.

[22] D.B. Williams, C.B. Carter, The Transmission Electron Microscope: A Textbook for Materials Science, Springer, 2009.

[23] M. Watanabe, D.B. Williams, The quantitative analysis of thin specimens: a review of progress from the cliff-lorimer to the new -factor methods, J. Microsc. 221 (2) (2006) 89-109. 
[24] J. Gregor, T. Benson, Computational Analysis and Improvement of SIRT, IEEE Trans. Med. Imaging 27 (7) (2008) 918-924.

[25] W. van Aarle, W.J. Palenstijn, J. De Beenhouwer, T. Altantzis, S. Bals, K.J. Batenburg, J. Sijbers, The ASTRA toolbox: a platform for advanced algorithm development in electron tomography, Ultramicroscopy 157 (2015) 35-47.

[26] A. Genc, L. Kovarik, M. Gu, H. Cheng, P. Plachinda, L. Pullan, B. Freitag, C. Wang, XEDS STEM tomography for 3D chemical characterization of nanoscale particles, Ultramicroscopy 131 (2013) 24-32.

[27] T.J.A. Slater, P.H.C. Camargo, M.G. Burke, N.J. Zaluzec, S.J. Haigh, Understanding the limitations of the Super-X energy dispersive $\mathrm{X}$-ray spectrometer as a function of specimen tilt angle for tomographic data acquisition in the S/TEM, J. Phys.: Conf. Ser. 522 (1) (2014) 012025.
[28] W. Van den Broek, A. Rosenauer, B. Goris, G. Martinez, S. Bals, S. Van Aert, D. Van Dyck, Correction of non-linear thickness effects in haadf stem electron tomography, Ultramicroscopy 116 (2012) 8-12.

[29] G. Lucas, P. Burdet, M. Cantoni, C. Hébert, Multivariate statistical analysis as a too for the segmentation of 3D spectral data, Micron 52-53 (2013) 49-56.

[30] C.L. Lawson, R.J. Hanson, Solving Least Squares Problems, SIAM, New Jersey, 1974.

[31] Z. Wang, A.C. Bovik, H.R. Sheikh, E.P. Simoncelli, Wavelets for image quality assessment: from error visibility to structural similarity, IEEE Trans. Image Process. 13 (4) (2004) 600-612.

[32] R.L. Streit, Poisson Point Processes: Imaging, Tracking, and Sensing, Springer Science \& Business Media, USA, 2010. 